\title{
Regenerative endodontic procedure combined with apical surgery of a necrotic permanent incisor with extensive periapical lesion using plasma rich in growth factors (PRGF): A Case report with 6 years post-op evaluation using CBCT
}

\author{
José-Francisco Gaviño-Orduña ${ }^{1}$, Inés Fernández-Guallart ${ }^{2}$, Javier Caviedes-Bucheli ${ }^{3}$, Manuel Espadas-García ${ }^{4}$, \\ José López-López ${ }^{5}$
}

\begin{abstract}
${ }^{1}$ DDs, PhD student. Dentist. Department of Odonto-Stomatology, School of Dentistry, University of Barcelona
${ }^{2}$ DDs, MsC Dentist. Department of Odonto-Stomatology, School of Dentistry, University of Barcelona

${ }^{3}$ DDs, MsC Centro de Investigaciones Odontologicas (CIO) Pontificia Universidad Javeriana Bogota Colombia

${ }^{4}$ MD, DDS, Dentist. Department of Odonto-Stomatology, School of Dentistry, University of Barcelona

${ }^{5} \mathrm{PhD}, \mathrm{MD}$, DDS, Dentist. University of Barcelona. Spain. Professor of Oral Patholoy. School of Dentistry, Barcelona University / Oral Health and Masticatory System Group (Bellvitge Biomedical Research Institute) IDIBELL, University of Barcelona, L'Hospitalet de Llobregat, Barcelona 08907, Spain
\end{abstract}

\section{Correspondence:}

Campus Universitario de Bellvitge

Pabellón de Gobierno, 2 planta

Feixa Llarga, s/n 08907

L'Hospitalet de Llobregat, Barcelona, España

dr.gavino@gmail.com

\begin{abstract}
Gaviño-Orduña JF, Fernández-Guallart I, Caviedes-Bucheli J, EspadasGarcía M, López-López J. Regenerative endodontic procedure combined with apical surgery of a necrotic permanent incisor with extensive periapical lesion using plasma rich in growth factors (PRGF): A Case report with 6 years post-op evaluation using CBCT. J Clin Exp Dent. 2021;13(6):e620-5.
\end{abstract}

Received: 08/12/202 Accepted: 23/01/2021

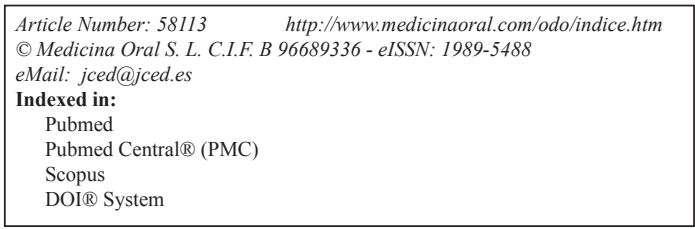

\begin{abstract}
Background: The aim of this case report is to describe the step-by step and outcomes of a treatment approach which simultaneously combines a Regenerative Endodontic Procedure (REP) and apical surgery using PRGF as treatment of a post-traumatic necrotic permanent central incisor with extensive periapical lesion and undeveloped apex in a 16 years-old patient.

Case description: A 16 years-old patient with an extensive periapical lesion in the maxillary central incisor was treated with a combination of REP and periapical surgery in the same visit. A Bi- antibiotic paste (BAP) was used to priorly disinfect the canal, which was posteriorly sealed with MTA placed over a PRGF clot at the same time that the periapical lesion was surgically debrided, removed and grafted, The radiographic and CBCT follow-up results showed a complete healing of the radiolucent lesion, which was replaced with bone-like tissue in two years and well preserved until six years later.

Practical implications: A combined approach of a Regenerative Endodontic Procedure and apical surgery using PRGF may be a good treatment modality in cases of extensive periapical lesions in necrotic teeth with open apexes. PRGF has shown to act as an ideal autologous matrix because it is stable, provides growth factors and bioactive molecules, and stimulates collagen production, angiogenesis, and cell differentiation.
\end{abstract}

Key words: Regenerative endodontic procedure, open apex necrotic tooth, periodontal apical lesion, growth factors, apical surgery. 


\section{Introduction}

Early dental trauma to immature teeth can result in loss of neurovascular supply, leading to interruption of rhizogenesis and the consequent weakening of the root structure, which can lead to fracture in $77 \%$ of the reported treated cases (1).

The choice between nonsurgical treatment and endodontic surgery is based on the benefits (capability to heal apical periodontitis) and risks associated to either alternative. This procedure combined with nonsurgical retreatment shows $1 \%-25 \%$ higher success rates than when endodontic surgery is performed alone (2).

Regenerative Endodontic Procedures (REPs) of necrotic immature teeth with open apexes have demonstrated predictable clinical results, since they provide three advantages over traditional methods of apexification in terms of root development: increasing root length, thickening root dentinal walls, and achievement of an apical closure (3). Disinfection of the root canal, obtaining a biological scaffold by achieving bleeding into the canal and sealing it with a biocompatible material seem to be key factors to provide the necessary environment for the success of REPs (3). In recent publications, Plasma Rich in Growth Factors (PRGF®-Endoret ${ }^{\circledR}$ ) used as a scaffold has shown the capability to stimulate collagen production, angiogenesis, and cell differentiation as well as anti-inflammatory and anti-bacterial properties both in REP and surgical treatment (4).
The aim of this case report is to describe the step-by step and outcomes of a single stage approach combining REP and apical surgery using PRGF as treatment of a post-traumatic necrotic permanent central incisor with extensive periapical lesion and undeveloped apex in a 16 years-old patient.

\section{Case Reports}

A 16-year-old male patient presented to the Department of Endodontics at the University of Barcelona referred by the Orthodontics Department. The patient had a non-remarkable medical history except for a traumatism occurred ten years before, in which he hit his front teeth. At the time, no dental treatment was performed, and since he did not have symptoms in the following years, he did not follow-up either. Upon examination during his orthodontics consultation, an asymptomatic extensive radiolucent lesion in the upper right maxilla was noticed in the panoramic X-ray (Fig. 1A).

The endodontic clinical examination revealed staining and an untreated enamel-dentin fracture on the maxillary right central incisor. The percussion test showed an augmented response for this tooth, while the cold vitality test (Endo Ice, Hygenic, Coltène/Whaledent Inc. Cuyahoga Falls, Ohio) was negative, being positive for the neighbouring teeth. No spontaneous pain was referred, and periodontal probing depths (CP-12, Hu-Friedy, Chicago, USA) were $2 \mathrm{~mm}$ all around the tooth, with no

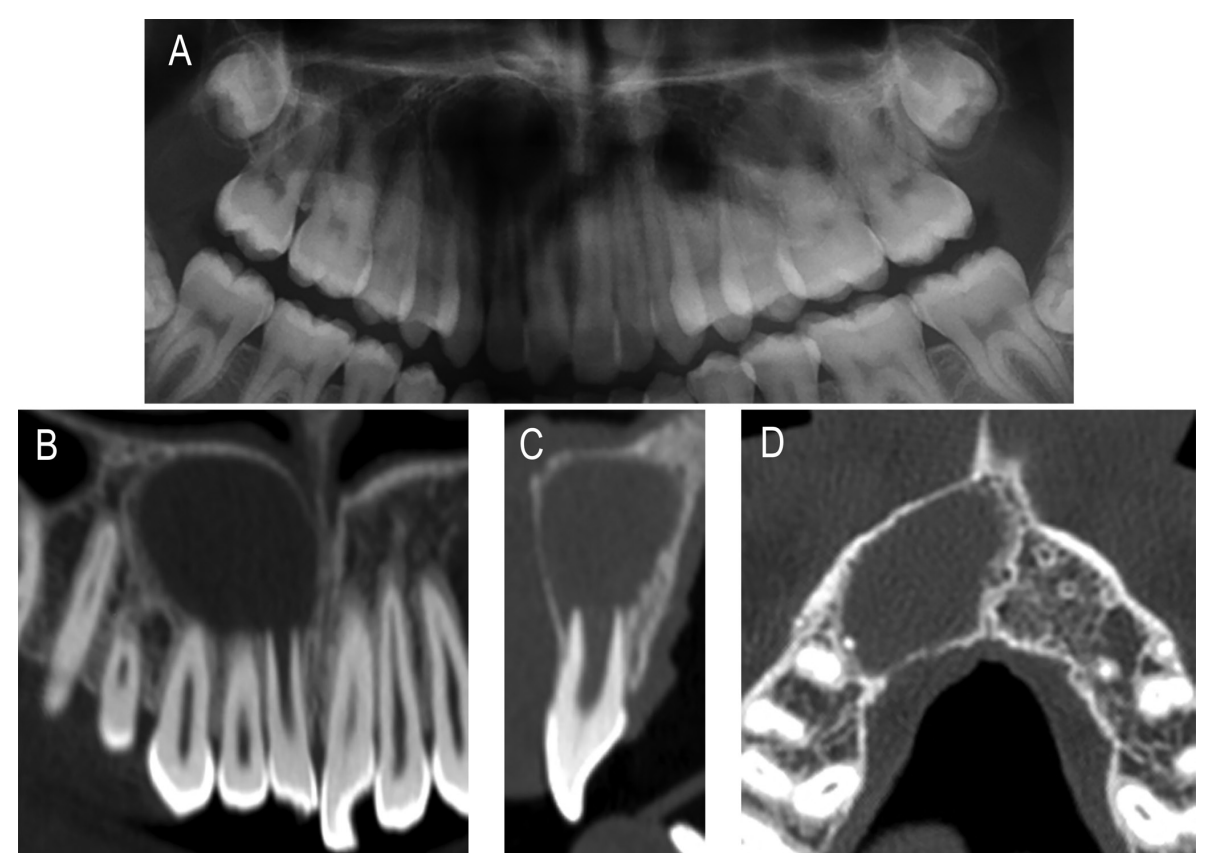

Fig. 1: A) Pre-op orthopantomography ilustrating a extensive radiolucent lesion in the upper right maxilla related to the apices of 1.1, 1.2, and 1.3. (B-D) Selected images of computed tomography B) Coronal slice showing the lesion affecting from 1.1 to $1.5 \mathrm{C}$ ) Sagital slice show the lesion in relation with open apex 1.1 D) Axial slice show extensive lesion occupying bone marrow in the upper right maxilla and bulging cortical bone plates. 
signs of mobility. A periapical radiograph (PA) using a paralleling technique revealed an incomplete root development showing an open apex for the right central incisor, corresponding to Nolla's eighth stage and associated with a large periapical radiolucency. The computed tomography evaluation (CBCT) confirmed the presence of an extensive periapical lesion $(19 \times 12 \times 16 \mathrm{~mm})$ and its intimate contact with the apexes of the maxillary right central and lateral incisors (Fig. 1B-D). A small fenestration was observed affecting the buccal bony wall of the maxillary right central incisor. Based on clinical and radiographic examinations, diagnosis of pulp necrosis and chronic apical periodontitis was established for this tooth. As it exhibited an open apex, REP with autologous Plasma Rich in Growth Factors (PRGF) was considered as a treatment option. As the periapical lesion was so extensive, it was decided to debride it simultaneously to the REP. The patient agreed, written informed consent was obtained and the treatment was conducted in accordance with ethical principles of the University of Barcelona Dental Hospital.

On the first appointment, a pulp chamber access was created. Minimal mechanical instrumentation was performed $2 \mathrm{~mm}$ above the apex with an ISO \#70 H-file, followed by copious irrigation with $5.25 \% \mathrm{NaOCl}$ solution. After drying the canal, a bi-antibiotic paste (BAP) consisting of a mixture of $250 \mathrm{mg}$ of metronidazole, $250 \mathrm{mg}$ of ciprofloxacin, and sterile water was introduced in the canal using a \#1 Buchanan hand plugger (SybronEndo, Orange, California, US) up to the apex. The access cavity was temporarily restored with $3 \mathrm{~mm}$ of Cavit ${ }^{\mathrm{TM}}$ (ESPE, Seefeld, Germany).

Two weeks later, during the second appointment, extraoral antisepsis was carried out using iodine solution, and local anaesthesia was administrated (articaine with epinephrine $40 \mathrm{mg} / \mathrm{ml}+0,01 \mathrm{mg} / \mathrm{ml}$ - Artinibsa, Laboratorios Inibsa S.A., Lliçà de Vall, Barcelona, Spain). A full thickness mucoperiosteal flap was reflected from a sulcular incision extended from distal of the maxillary right canine to distal of the maxillary left central incisor. A large periapical bone defect associated with partial loss of the buccal cortical plate of the right central and lateral incisors was encountered. This fenestration was extended using a tungsten round bur 023 (Komet, Lemgo, Germany). The lesion was circumferentially separated from the bony crypt and the affected teeth using a 10 miller surgical curette (Hu-Friedy, Chicago, USA). Once removed, it was measured (11 $\mathrm{mm} \times 8 \mathrm{~mm} \times 12$ $\mathrm{mm}$ ), fixed in $10 \%$ buffered formalin, and sent for histopathological examination. The surgical area was then rinsed with sterile saline solution. When the apical involvement of the lateral incisor was confirmed, a conventional endodontic treatment was performed, and its apex was resected, followed by the retrograde placement of a Mineral Trioxide Aggregate (MTA) apical barrier (Dentsply Tulsa Dental, Johnson City, TN).
With the flap raised and the apex exposed, the root canal of the central incisor was irrigated with sterile saline to remove the BAP, which was carefully collected with a surgical suction system positioned through the fenestration in the apex area. In a similar fashion, the canal was then rinsed with $5.25 \% \mathrm{NaOCl}$ solution from coronal to apical. After complete disinfection, a final irrigation with sterile saline was performed.

Plasma rich in growth factors (PRGF®-Endoret $\left.{ }^{\circledR}\right)$ was prepared according to the manufacturer's instructions (BTI Biotechnology Institue S.L, Vitoria, Spain): Then, the plasma column was divided into two fractions: fraction 2 (F2), defined as the $2 \mathrm{~mL}$ of plasma just above the buffy coat, and fraction 1 (F1), defined as the plasma column above the F2. This gave a total of $8 \mathrm{~mL}$ of F2. The volume of F1 would depend on the haematocrit value of the patient.

The PRGF was used in the REP as follows: $2 \mathrm{~mL}$ of inactivated F2 was injected in the apical third of the root canal using a sterile syringe. The remaining $6 \mathrm{~mL}$ were gently poured in a sterile glass container and activated by adding $300 \mu \mathrm{L}$ of $10 \%$ calcium chloride solution, which triggered the formation of a three-dimensional fibrin clot as well as the release of growth factors and proteins from autologous platelets (Fig. 2A). This clot was divided in two: the first $3 \mathrm{ml}$ clot was used to fill the apical third of the root canal from the surgical access until 3 millimetres below the cementoenamel junction (CEJ), occupying both the apical foramen and the periapical tissues (Fig. 2B,C); the other $3 \mathrm{ml}$ clot was mixed with 1cc Tri-calcium phosphate (B-TCP) (Isios, Kasios, l'Union, France), generating a new sticky bone that was used to fill the bone defect (Fig. 2D,E). The activated F1, kept at $37{ }^{\circ} \mathrm{C}$ to obtain a haemostatic and elastic fibrin membrane, was placed over the bone graft, acting like a membrane by closing the entire defect (Fig. 2F). The mucoperiosteal flap was repositioned and held in place using 4-0 non-absorbable surgical sutures (Supramid, Aragó, Barcelona, Spain).

MTA was mixed with saline solution and placed inside of the root canal from an orthograde way, on the top of the PRGF and until $1 \mathrm{~mm}$ below the cemento-enamel junction (CEJ) to prevent crown staining. The F1 fibrin membrane's consistency allowed to place the MTA safely as it permitted the total control of its final position. A moistened cotton pellet was placed in the pulp chamber, and $3 \mathrm{~mm}$ of Cavit ${ }^{\mathrm{TM}}$ (ESPE, Seefeld, Germany) sealed the access cavity. A postoperative radiography was taken. The patient was kept under antibiotic and analgesic coverage. Chlorhexidine gluconate solution $(0.2 \%)$ was prescribed as a mouth rinse for a period of five days. After a week, the suture was removed, and as the healing was uneventful, the final restoration was performed using an adhesive composite. The previously excised tissue sent for histopathological examination was diagnosed as Pe- 


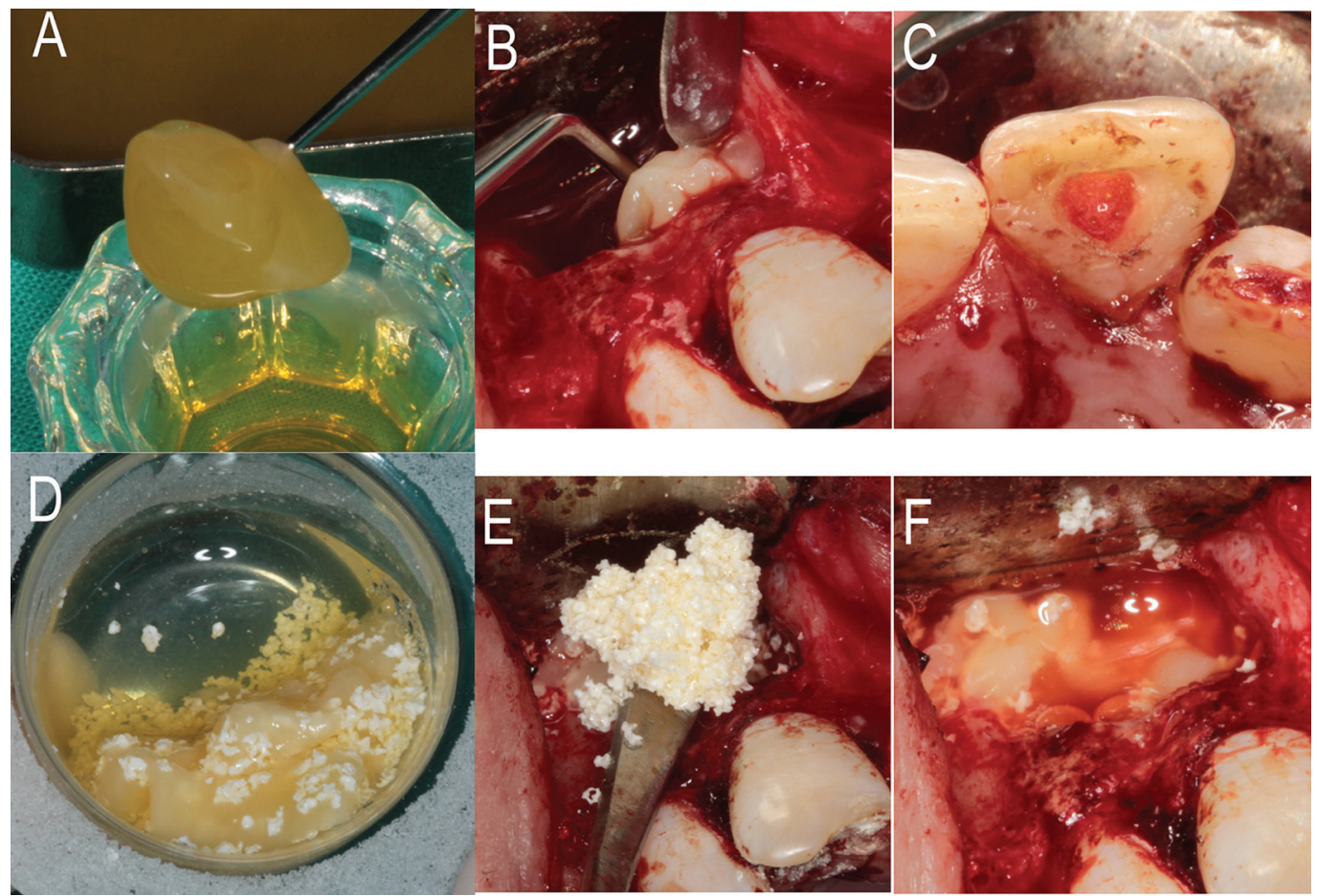

Fig. 2: Intra-op clinical photographs of use of PRGF A) Three-dimensional clot obtained from F2 B) Filling the apical third of the root canal with the F2 clot. C) F2 clot filling the canal until 3 millimeters below the cementoenamel junction. D) Three-dimensional clot obtained by mixing F2 with (B-TCP) E) Clot of F2 and (B-TCP) filling intrabony defect. F) Fibrin membrane from F1 closing all defect.

riapical Granuloma. In the follow-up visits at 3, 6, 12 and 24 months, there were no symptoms of pain, discomfort or inflammation. $\mathrm{CBCT}$ at 24 months showed the complete disappearance of the extensive apical lesion and its replacement with new bone-like tissue, thus confirming the satisfactory healing of the defect and the overall treatment successful outcome (Fig. 3A,C). CBCT at a 6-years follow-up confirmed the stability of the results and the slight ingrowth of bone-like tissue inside of the root canal space in the apical portion of the tooth, a slightly increase of root length was observed too (Fig. 3B,D,E).

\section{Discussion}

-Root canal disinfection protocol

Historically other methods such apexification using $\mathrm{Ca}$ $(\mathrm{OH}) 2$ have been used to treat necrotic immature teeth with open apexes, but the literature shows that REP provides significantly greater tooth survival $100 \%$ versus $77 \%$ (3). Many of these failures are cervical fractures due to weakness caused by long-term use of calcium hydroxide (5). In the present case, the disinfection protocol of the root canal is based on a copious sodium hypochlorite irrigation combined with BAP as intracanal medicament. High (5-6\%) NaOCl concentrations have been used in $36 \%$ of the published cases of REPs (6). There are reported cases of crown discoloration as a side effect after applying a triple antibiotic paste (TAP) made by ci- profloxacin, metronidazole, and minocycline, which are caused by the last one (7). This confirms the indication of using BAP instead of TAP in the REP protocol.

-PRGF use both in REP and GBR

In the reported case, PRGF was used both as an intrapulpal and intrabony matrix as well as like a membrane to prevent epithelial migration. In REP, a matrix is necessary to provide a physiochemical and biological microenvironment that supports the growth, migration, and differentiation of stem cells (8). PRGF is versatile, easy to prepare, and can be used as a matrix both for pulp revascularization and bone regeneration. Firstly, it provides conduction: creating a three-dimensional fibrin scaffold that retains part of the released protein content, maintains the regenerative space and works as a matrix for endogenous cells. Moreover, this matrix allows the correct placement of MTA at the optimal level (8). Secondly, it provides induction, supplying growth factors and bioactive molecules which are able to influence recruitment, growth, and morphogenesis of cells in order to promote the repair of the tooth's pulp system in REP and regeneration of bone in apical surgery $(4,9)$.

Furthermore, in REP, platelet-rich plasma (PRP) has shown a better clinical outcome in terms of periapical healing, apical closure, and root dentinal wall thickening when compared with a blood clot and PRP is the first choice when the blood clot is insufficient, or no bleeding 

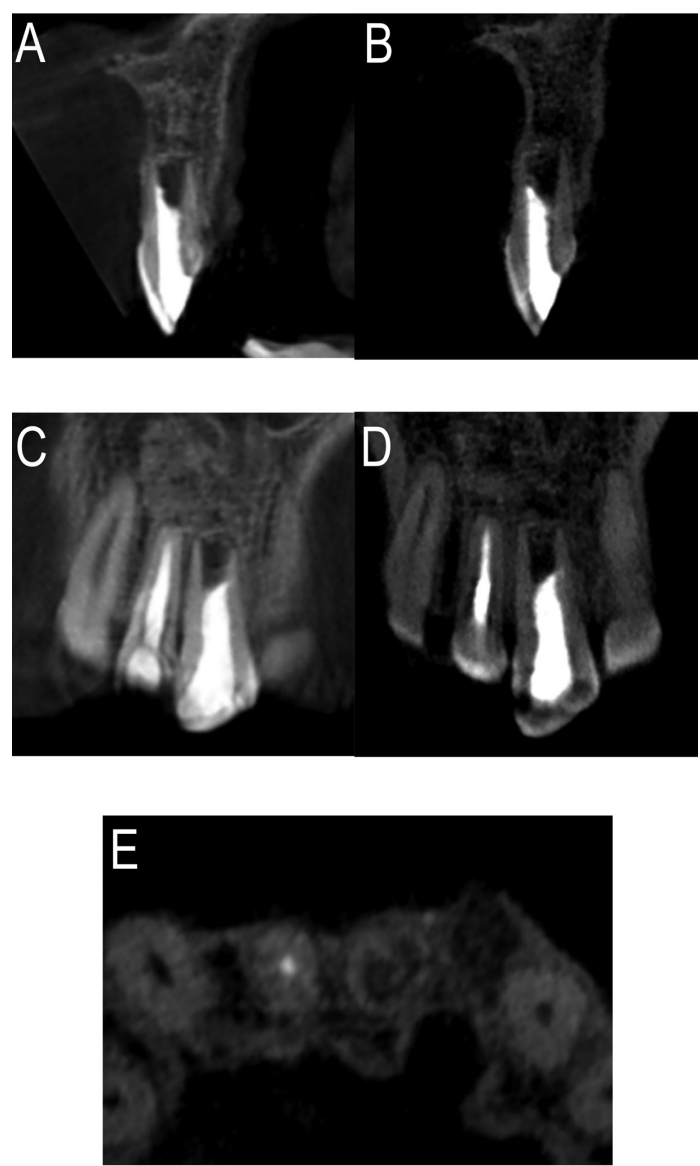

Fig. 3: Images of cone-beam computed tomographic scan visualized by Planmeca Romexis Viewer v. 3.4.1.R. at two years (A and C) and Kodak Dental Imaging Software 3D Module v 2.4 at six years (B, D and E). A and B) Sagital slice at 1.1 level at two and six years. C and D) Coronal slice at two and six years. E) Axial slice at six years. All of them show the complete resolution of the extensive apical lesion and bone regeneration, with a slight ingrowth of bone-like tissue inside of the root canal space in the apical portion of the tooth and a slightly increase of root length at 6-years follow-up.

is found when irritating the apical tissue (10). In the present case, the size of the periapical lesion did not allow for over-extensive penetration of the canal with an endodontic file with the goal of stimulating bleeding. In this case, PRGF allowed for a sufficient and competent scaffold as well as a functional support to place the MTA. -Guided Bone Regeneration

Guided Bone Regeneration (GBR) techniques are needed to avoid the defect healing by fibrous connective tissue when it is too extensive (9). The combination of PRGF and Beta-tricalcium phosphate ( $\beta$-TCP) as bone graft has been used in this case. Firstly, PRGF provides a provisional matrix for the attachment and migration of immune cells, fibroblasts, endothelial cells, and tissue cells. Secondly, the bone graft, which exhibits osteoconductive capability, serves as a scaffold on which cells can attach, mi- grate, grow, and divide. The addition of PRP to bone graft facilitates a better and faster bone regeneration compared to PRP alone (11). In addition, the use of PRGF decreases postoperative discomfort, reducing the most common inflammatory symptoms such as pain and swelling (12). When PRP is added to tricalcium phosphate $8 \%$ to $10 \%$, more vital bone formation is obtained (13).

-Success of the case

In the REP literature, it has been suggested that the success in promotion of root development, thickening of the root dentinal walls, and apical closure is related to the existence of stem cells from the apical papilla (SCAP), which exist even in necrotic teeth. Their potential of self-renewal and differentiation into chondroblasts, osteoblasts, adipocytes and functional dentinogenic cells has been confirmed (14). In the present case, no mineralized tissue growth was obtained on the tooth walls or in the pulp space at two years, and a slight ingrowth of bone-like tissue from the periapical areas was observed after 6 years. As the chances of having the Hertwig's epithelial root sheath maintained are very low, the PDL stem cells (a reservoir of multipotent postnatal stem cells present in adults (15)) could have been responsible for the healing pattern showed in terms of tissue repair. The CBCT findings (i.e., complete disappearance of the radiolucent lesion, wound healing, solving of the fenestration, and even the tooth canal's filling with a bone-like material), combined with the absence of clinical signs and symptoms after 2 years, and maintained after 6 years, indicate the success of the procedure.

This case report proposes a single stage procedure combining REP therapy and apical surgery using PRGF as treatment of a tooth that would otherwise have had a long and complex management, with several visits separated months from one another. Within the limitations of this clinical case, a combined approach may be a good treatment modality in cases of extensive periapical lesions present in necrotic teeth with open apexes. However, further research under strict controlled conditions is needed to validate these results.

\section{References}

1. Andreasen FM, Kahler B. Pulpal response after acute dental injury in the permanent dentition: Clinical implications - A review. J Endod. 2015;41:299-308.

2. Torabinejad M, Corr R, Handysides R, Shabahang S. Outcomes of nonsurgical retreatment and endodontic surgery: a systematic review. J Endod. 2009;35:930-7.

3. Jeeruphan T, Jantarat J, Yanpiset K, Suwannapan L, Khewsawai P, Hargreaves KM. Mahidol study 1: Comparison of radiographic and survival outcomes of immature teeth treated with either regenerative endodontic or apexification methods: A retrospective study. J Endod. 2012;38:1330-6.

4. Anitua E, Alkhraisat MH, Orive G. Perspectives and challenges in regenerative medicine using plasma rich in growth factors. J Control Release. 2012;157:29-38.

5. Valera MC, Albuquerque MTP, Yamasaki MC, Vassallo FN, da Silva DA, Nagata JY. Fracture resistance of weakened bovine teeth after long-term use of calcium hydroxide. Dent Traumatol. 2015;31:385-9. 
6. Kim SG, Malek M, Sigurdsson A, Lin LM, Kahler B. Regenerative endodontics: a comprehensive review. Int Endod J. 2018;51:1367-88.

7. Kim JH, Kim Y, Shin SJ, Park JW, Jung IY. Tooth discoloration of immature permanent incisor associated with triple antibiotic therapy: A case report. J Endod. 2010;36:1086-91.

8. Gaviño Orduña JF, Caviedes-Bucheli J, Manzanares Céspedes MC, Berástegui Jimeno E, Martín Biedma B, Segura-Egea JJ, et al. Use of Platelet-rich Plasma in Endodontic Procedures in Adults: Regeneration or Repair? A Report of 3 Cases with 5 Years of Follow-up. J Endod. 2017;43:1294-301.

9. Tsesis I, Rosen E, Tamse A, Taschieri S, Del Fabbro M. Effect of guided tissue regeneration on the outcome of surgical endodontic treatment: A systematic review and meta-analysis. J Endod. 2011;37:103945.

10. Zhang DD, Chen X, Bao ZF, Chen M, Ding ZJ, Zhong M. Histologic comparison between platelet-rich plasma and blood clot in regenerative endodontic treatment: an animal study. J Endod. 2014;40:138893.

11. Vaishnavi C, Mohan B, Narayanan LL. Treatment of endodontically induced periapical lesions using hydroxyapatite, platelet-rich plasma, and a combination of both: An in vivo study. J Conserv Dent. 2011;14:140-6.

12. Del Fabbro M, Ceresoli V, Lolato A, Taschieri S. Effect of platelet concentrate on quality of life after periradicular surgery: A randomized clinical study. J Endod. 2012;38:733-9.

13. Wiltfang J, Schlegel KA, Schultze-Mosgau S, Nkenke E, Zimmermann R, Kessler P. Sinus floor augmentation with $\beta$-tricalciumphosphate $(\beta-\mathrm{TCP})$ : Does platelet-rich plasma promote its osseous integration and degradation? Clin Oral Implants Res. 2003;14:213-8.

14. Abe S, Yamaguchi S, Watanabe A, Hamada K, Amagasa T. Hard tissue regeneration capacity of apical pulp derived cells (APDCs) from human tooth with immature apex. Biochem Biophys Res Commun. 2008:371:90-3.

15. Seo BM, Miura M, Gronthos S, Bartold PM, Batouli S, Brahim $\mathrm{J}$, et al. Investigation of multipotent postnatal stem cells from human periodontal ligament. Lancet. 2004;364:149-55.

\section{Authors' contributions}

José F. Gaviño Orduña: Performed the clinical treatment. Drafted the article. Final approval of the version to be submitted.

Inés Fernández Guallart: Performed the clinical treatment. Drafted the article. Final approval of the version to be submitted

Javier Caviedes Bucheli: Revised the article critically for important intellectual content. Final approval of the version to be submitted. Manuel Espadas: work in platelet concentrates, Drafted the article. Final approval of the version to be submitted.

José López López: Revised the article critically for important intellectual content. Final approval of the version to be submitted.

\section{Source of Funding}

No funding was needed.

\section{Ethics}

No ethic committee was needed.

\section{Conflict of interest}

The authors declare that they don't have any conflict of interest related to this article. 